\title{
The Influence of Scaling and Assortativity on Takeover Times in Scale-Free Topologies
}

\author{
Joshua L. Payne \\ Dept. of Computer Science \\ University of Vermont \\ Burlington, VT 05405 \\ 802-656-9116 \\ Joshua.Payne@uvm.edu
}

\author{
Margaret J. Eppstein \\ Dept. of Computer Science \\ University of Vermont \\ Burlington, VT 05405 \\ 802-656-1918 \\ Maggie.Eppstein@uvm.edu
}

\begin{abstract}
In evolving systems, the topological characteristics of population structure have a pronounced impact on the rate of spread of advantageous alleles, and therefore affect selective pressure. One common method for quantifying the influence of population structure on selective pressure is through the analysis of the expected number of generations required for a single favorable allele to saturate an entire population (a.k.a. takeover time analysis). While takeover times have been thoroughly investigated in regular population structures, the selective pressures induced by irregular interaction topologies, such as scale-free graphs, have received much less attention. In this study, we systematically investigate the influence of scaling and assortativity, two frequently overlooked topological properties, on takeover times in scale-free population structures. Our results demonstrate that the scaling parameter and the magnitude and sign of assortativity have profound and unexpected nonlinear influences on takeover times in scale-free interaction topologies. We explore the reasons behind these results and suggest ways in which they may be exploited in future studies.
\end{abstract}

\section{Categories and Subject Descriptors}

I.2.8 Artificial Intelligence [Problem Solving, Control Methods and Search]: Heuristic Methods

\section{General Terms}

Algorithms, Performance, Design, Experimentation

\section{Keywords}

Assortativity, Interaction Topologies, Mixing Patterns, Population Structure, Saturation Dynamics, Scale-free, Spatial Structure, Takeover Time, Selective Pressure

Permission to make digital or hard copies of all or part of this work for personal or classroom use is granted without fee provided that copies are not made or distributed for profit or commercial advantage and that copies bear this notice and the full citation on the first page. To copy otherwise, or republish, to post on servers or to redistribute to lists, requires prior specific permission and/or a fee.

GECCO'08, July 12-16, 2008, Atlanta, Georgia, USA

Copyright 2008 ACM 978-1-60558-130-9/08/07 ...\$5.00.

\section{INTRODUCTION}

The behavior of complex adaptive systems is governed by the collective dynamics of the interacting system components. Consequently, the topological characteristics of the underlying interaction network strongly influence the rate of flow of information throughout the system, and thus play a critical role in determining emergent system-wide dynamics. The structural properties of graph-based interaction networks have been shown to have a pronounced impact on important phenomena, such as the evolution of cooperative behavior in social dilemmas [11][19][25], the emergence of global cascades in models of binary decisions with externalities [29], the maintenance of genetic diversity in simulated evolving populations [27], the rate of epidemiological invasions [13][20], and the reduction of selective pressure in evolutionary algorithms [7][24][26]. Thus, whether studying the dissemination of innovative ideas or the proliferation of advantageous mutations, it is important to understand the influence of the underlying interaction network on the flow of information throughout the system.

In evolving systems, one common technique for quantifying how the flow of information is influenced by a given interaction topology is through the analysis of takeover time [8], which is defined as the expected number of generations required for a single advantageous allele to fully saturate a population, in the absence of variation operators such as recombination and mutation. Higher takeover times imply lower selective pressure, and vice versa. While takeover time analysis has typically been utilized to understand the influence of population structure on the saturation dynamics of evolutionary algorithms, it can also serve as a simplified measure of information flow in general, and so can provide a baseline for the analysis of a variety of graph-based models of dynamical processes.

Takeover dynamics have been previously investigated and modeled in several regular (constant degree) population structures [7][8][24][26], as well as random and small-world topologies [6]. The general result of these studies is that regular (or nearly regular) lattice structures reduce selective pressure, relative to well-mixed or randomly mixed populations, and can thus enhance the exploratory power of evolutionary search. However, takeover time analysis in "scale-free" topologies [3] has received considerably less attention, despite the fact that many real-world interaction networks fall into this class of graphs [1][5][12][15]. In scale-free topologies, the probability $p(k)$ of finding a vertex of degree $k$ is of the form $p(k) \propto k^{-\gamma}$, where $\gamma$ is referred to as the scaling parameter. The heterogeneity of scale-free topologies, 
captured in part by $\gamma$, has been shown to have an important influence on several important dynamical processes, ranging from percolation [9] to the emergence of cooperation in evolutionary games [22]. Some scale-free topologies (e.g., societal interaction networks) exhibit "assortative mixing" [18]; i.e., vertices of high degree are more likely to be attached to one another than to vertices of low degree. In contrast, certain biological networks, such as protein-protein interactions and ecological food webs exhibit "disassortative mixing" [18], where nodes of high degree are more likely to attach to nodes of low degree. Similar to the effects of the scaling parameter $\gamma$, the assortativity of scale-free topologies has also been shown to have a pronounced impact on dynamical processes occurring on these networks [18][23][30]. To date, only two studies [6][21] have analyzed takeover dynamics in scale-free topologies, and neither have examined the effects of the scaling parameter or the assortativity. In [6], Giacobini et al. investigated takeover dynamics on scale-free topologies generated using the algorithm provided by Albert and Barabási (AB) [3]. This scale-free graph generating algorithm yields a scaling parameter that approaches $\gamma \sim 3$ as the size of the graph approaches infinity and possesses an uncorrelated mixing pattern [18] (i.e., the mixing pattern is neither assortative nor disassortative). The results of [6] showed that the selective pressures induced by $\mathrm{AB}$ scale-free population structures are at least as strong as those induced by panmictic interaction topologies and that takeover time can be dramatically reduced by strategically placing the initial copy of the high fitness individual in a highly connected vertex. Using a variety of scale-free graph generating algorithms, Payne and Eppstein [21] showed that the selective pressures induced by scale-free interaction topologies with various degrees of clustering, modularity, and hierarchical organization, are heavily influenced by underlying topological properties and, by manipulating these characteristics, selective pressures on scale free graphs can be tuned from very high levels, such as those induced by random mixing, to low levels that are even weaker than those induced by nearest neighbor interactions. Their results also suggest that it may be possible to rapidly estimate takeover times on scale-free population structures based on only a few statically computed metrics of the underlying topology, such as the maximum path length and the variance in the distribution of path lengths.

Understanding dynamics on scale-free interaction networks may provide insights into numerous dynamical processes, as well as help to determine the potential for exploiting these properties in evolutionary computation. Consequently, the focus of this study is to analyze the independent and combined effects of scaling and assortativity on takeover times in scale-free population structures.

\section{METHODS}

\subsection{Definitions and Metrics}

The population structure of an evolutionary algorithm can be represented as a graph $G=(V, E)$, defined as a nonempty finite set of vertices $(V)$ and a finite set of edges $(E)$ connecting these vertices. Each individual in the population is represented by a vertex $i \in V$, so that $|V|=\mu$, where $\mu$ is the population size. The graph is undirected, with an edge $<i, j>\in E$ for every individual $j$ in the mating neighborhood of individual $i$, for all $i \in V$. In this study, we consider the simple case of a population with only two levels of fitness. The initial population comprises a single high fitness individual and $\mu-1$ low fitness individuals. If $N_{t}$ denotes the proportion of high fitness individuals at time $t$, then the takeover time of an individual experiment $T=\min \left\{t \mid N_{t}=1\right\}$ is defined as the minimum number of generations such that copies of the most fit individual fully saturate the entire population, starting with only one such individual in the initial population [24]. $E_{i}[T]$ is defined as the empirical estimate of the expected takeover time given that the initial best individual is located in vertex $i$, and the overall empirically estimated takeover time $E[T]$ is computed as the average of $E_{i}[T]$ over all vertices $i \in V$.

In order to most directly infer the influence of scaling and assortativity on takeover times in scale-free topologies, we adopt a simple "replace if better" selection mechanism (a.k.a. uniform selection [10]), where nodes are updated synchronously. For each node $i \in V$, a node $j$ is selected at random from the mating neighborhood of node $i$, with neighborhood size $k_{i}$. Thus, if there are $x$ nodes of high fitness in the mating neighborhood of node $i$, then the probability of selecting one of them is simply $x / k_{i}$ and the value of node $j$ replaces the value of node $i$ if node $j$ has higher fitness.

The distribution of vertex connectivity, $p(k)$, is a probability distribution function (PDF) depicting the frequency with which nodes have degree $k$. The complementary cumulative distribution function (CCDF), commonly used to visualize scale-free distributions [4], depicts the frequency with which nodes have degree greater than $k$. For all of the graphs considered in this study, the PDF of vertex connectivity will take the form $p(k) \propto k$ $\gamma$, where the scaling parameter, $\gamma$, will be systematically varied. Note that the resulting CCDF is then of the form $P(k) \propto k^{-\gamma+1}$. The path length $L_{i}$ of a vertex $i$ is defined as the mean of the shortest paths between $i$ and all of the other vertices in the graph. The maximum of the individual path lengths of all nodes is referred to as $L_{M A X}$. The characteristic path length $L$ of a graph $G$ is the average of the path lengths of the individual vertices $L_{i}$, with variance $\sigma_{\mathrm{L}}{ }^{2}$. The metric suggested in [21] to predict takeover times in spatially structured populations is defined as follows:

$$
\zeta=L_{M A X}+L_{M A X} \sigma_{L}^{2}
$$

The assortativity $r$ of a graph $G$ is a measure of the propensity with which vertices of similar degree connect to one another. Formally, assortativity $(r)$ is defined as [18]:

$$
r=\frac{|E|^{-1} \sum_{\forall<i, j>\in E} k_{i} k_{j}-\left[|E|^{-1} \sum_{\forall<i, j>\in E} \frac{1}{2}\left(k_{i}+k_{j}\right)\right]^{2}}{|E|^{-1} \sum_{\forall<i, j>\in E} \frac{1}{2}\left(k_{i}^{2}+k_{j}^{2}\right)-\left[|E|^{-1} \sum_{\forall<i, j>\in E} \frac{1}{2}\left(k_{i}+k_{j}\right)\right]^{2}}
$$

where $k_{i}$ and $k_{j}$ are the degrees of the nodes at the ends of an edge $<i, j>$. A graph is said to be assortative if $r>0$, uncorrelated if $r=$ 0 , and disassortative if $r<0$.

\subsection{Tuning the Scaling Exponent}

The growing random network (GN) model of Krapivsky, Redner, and Leyvraz [14] can be used to generate scale-free topologies with a range of scaling parameters. Briefly, the GN algorithm works as follows. In each time step $t$, a single node is added to the graph, connecting to an existing node of degree $k$ with probability proportional to the linear connection kernel $A_{k}$, where $A_{k}=1$ if $k=$ 1 and $A_{k}=\alpha k$ if $k>1$. By altering the parameter $\alpha$ in the connection kernel, the scaling parameter of the degree distribution 
can theoretically be tuned anywhere in the range $2<\gamma<\infty$, for infinitely sized graphs [14]. However, due to finite size effects, we restricted our investigation to scaling parameters in the range $2.4 \leq \gamma \leq 4$. Note that if $\alpha=1$, the AB algorithm [3] is recovered.

We modified the GN algorithm slightly, so that instead of initializing the graph as a single node, we initialize it with a clique of $m_{0}$ nodes with no edges, as in [21]. Newly arriving nodes are connected to $m\left(=m_{0}\right)$ existing nodes, as opposed to simply making a single connection. After $t$ time steps, the graph comprises $t+m_{0}$ nodes and $m t$ edges. Since we set $m=m_{0}$, graph connectivity is ensured. In order to obtain finite-sized graphs with specific scaling parameters, graph instances were created repeatedly and for each the observed $\gamma$ was calculated as described in the next paragraph. Only those graphs with observed scaling parameters within 0.01 of the desired scaling parameter were saved and used in the experiments.

In order to accurately estimate the scaling parameter $(\gamma)$ and lower bound $\left(k_{\min }\right)$ of the scale-free region of the degree distribution, we utilized the method provided by Clauset, Shalizi, and Newman [4]. By varying $\gamma$ and $k_{\text {min }}$ over a specified range and iteratively applying the Kolmogrorov-Smirnov (KS) test to the observed data and fitted model, the combination of $\gamma$ and $k_{\min }$ that minimized the KS statistic was chosen as the hypothesized power-law model. The goodness of fit of this model was then calculated using a Monte-Carlo procedure [4] in order to verify that the degree distribution of the resulting graph was, in fact, consistent with the hypothesized power-law model.

In order to eliminate any structural motifs that may have been inadvertently introduced into the topology as an artifact of the graph construction algorithm, we then randomized the edge set of each saved topology using the Maslov-Sneppen algorithm [16], as follows. In each step of the algorithm, two edges, $\langle a, b\rangle$ and $<c, d>$, are chosen at random with uniform probability. Their connections are then swapped such that two new edges, $\langle a, c\rangle$ and $\langle b, d\rangle$ replace the original pair of edges. If either of the newly formed edges are already present in the graph, then the edge swap is aborted. This process is repeated for 10,000 edge swaps. Since the degree of each node remains unchanged after a swap, this method exactly preserves the underlying degree distribution.

\subsection{Tuning the Assortativity}

Xulvi-Brunet and Sokolov [30] have proposed a single-parameter shuffling algorithm that probabilistically alternates between Maslov-Sneppen edge swaps, and edge swaps that alter assortativity. However, since the assortative edge swaps are performed probabilistically, their algorithm yields a range of assortativities for a given specified probability. In this study, we wanted to investigate takeover times on topologies with wellcontrolled assortativities, so we devised the following iterative method that allows for the direct specification of assortativity, within some error tolerance.

In each iteration of our algorithm, the assortativity of the graph is measured using equation (2) and compared to the desired assortativity. Two edges, $\langle a, b\rangle$ and $\langle c, d\rangle$, are then selected at random with uniform probability. If the observed assortativity is less than the desired assortativity, the edges are swapped such that the two nodes with the larger degrees are connected together and the two nodes with the smaller degrees are connected together. If the observed assortativity is greater than the desired assortativity, the reverse is done. If either of the new edges are already present in the graph, then the swap is aborted. The algorithm iteratively continues swapping edges in this fashion until the observed assortativity is within some epsilon of the desired assortativity. If the desired assortativity is not achieved within 10,000 swaps, the graph is discarded. Since vertex degree is never altered in a swapping event, this algorithm exactly preserves the underlying degree distribution.

\subsection{Experimental Design}

A population size of $\mu=1024$ was used in all experiments. Scalefree topologies with scaling parameters $\gamma \in\{2.4,2.6,2.8,3.0$, 3.2, 3.4, 3.6, 3.8, 4.0 $\}$ were generated, as described in Section 2.2. Once graphs with the desired scaling parameters were obtained, they were subsequently shuffled as described in Section 2.3 to have assortativities $r \in\{-0.2,-0.1,0,0.1,0.2\}$. In all cases, $m_{0}=$ $m=2$, such that the average degree $<k>=4$. The goodness of fit for each hypothesized power-law model was found to be satisfactory $(p>0.1$, using the Monte Carlo method in [4]) and $k_{\min }$ was found to range from 3 to 5 . Ten graph instances were generated for each combination of $\gamma$ and $r$, using these methods. If any graph became disconnected during shuffling, then that graph instance was discarded, a new graph was generated, and the process was repeated.

In contrast to regular population structures, the expected takeover times in scale-free population structures have been shown to be highly dependent upon the placement of the initial copy of the high fitness individual [6]. Therefore, for each graph instance we systematically placed the high fitness individual of the initial population in each node of the topology, one at a time, and subsequently performed ten independent takeover time simulations for each initial placement, in order to mitigate the stochasticity inherent in the selection policy. Thus, 102,400 independent runs were performed for each combination of $\gamma$ and $r$, resulting in a total of 4,608,000 independent takeover time simulations.

\section{RESULTS AND DISCUSSION}

\subsection{Influence of Scaling Alone}

We first consider the case of uncorrelated $(r=0)$ scale-free topologies. Takeover times of the individual simulations $(T)$ are shown as a function of the scaling parameter $(\gamma)$ in Figure 1a, with average takeover times $(E[T])$ provided in Figure 1b. In Figure 1c, we depict the average takeover time given that the initial high fitness individual is placed in node $i\left(E_{i}[T]\right)$, as a function of the degree of this initial vertex $\left(k_{i}\right)$. I.e., each data point in Figure 1c represents the average takeover time observed across all simulations in which the vertex of initial placement had degree $k_{i}$. The relationship between $E_{i}[T]$ and $k_{i}$ appears to decay according to a power-law, implying that $E_{i}[T]$ decreases faster than linearly in $k_{i}$. As evidenced in Figure $1 \mathrm{c}$, the scaling parameter $(\gamma)$ of the population structure affects the relationship between $E_{i}[T]$ and $k_{i}$. $E[T]$ decreased from 34.8 generations to 22.7 generations as the scaling parameter $(\gamma)$ increased from $\gamma \sim 2.4$ to $\gamma \sim 4.0$ (Figure 1b) and the standard deviation of $E[\mathrm{~T}]$ across graph instances similarly decreased from 1.58 for $\gamma \sim 2.4$ to 0.05 for $\gamma \sim 4.0$. The decrease in $E[T]$ occurs for two reasons, as described below.

First, scale-free topologies with lower scaling parameters possess more highly connected vertices than those with larger scaling 

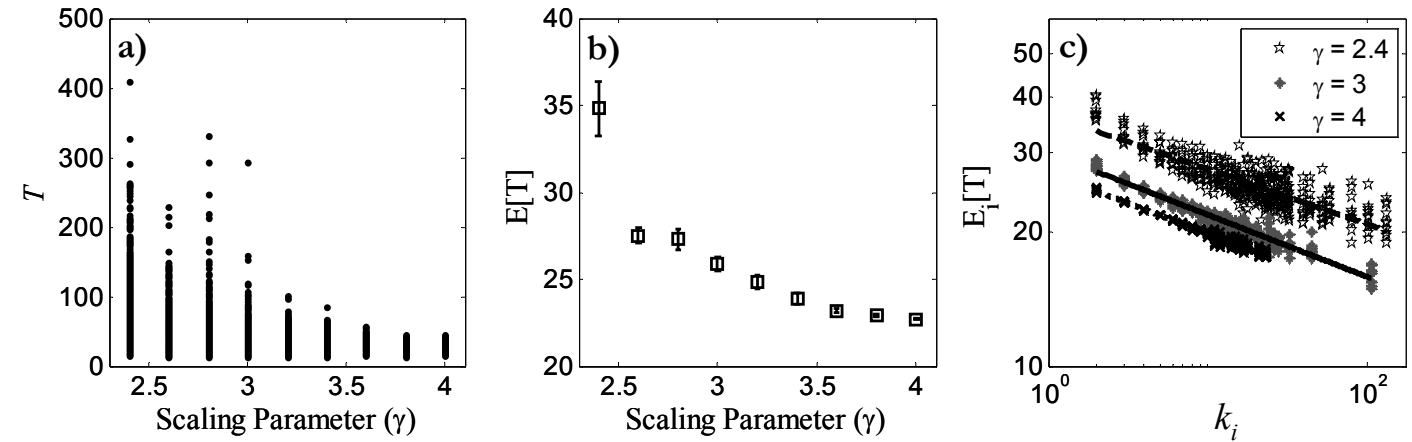

Figure 1. Influence of scaling on takeover times (in generations) in uncorrelated $(r=0)$ scale-free topologies. (a) Individual takeover times $(T)$ of all independent simulations are presented as a function of the scaling parameter $(\gamma)$. (b) Average takeover times $(E[T]$, averaged over all simulations on each of 10 graph instances, error bars denote one standard deviation) are depicted as a function of the scaling parameter $(\gamma)$. (c) Average takeover times given that the initial high fitness individual was placed in vertex $i\left(E_{i}[T]\right)$ are shown as a function of the degree of the initial vertex $\left(k_{i}\right)$. The dashed $(\gamma \sim 2.4)$, solid $(\gamma \sim 3.0)$, and dotted $(\gamma \sim 4.0)$ lines are included as a visual aid to indicate trends as a function of scaling and $k_{i}$. Note the change in scale in the vertical axes between (a) and (b). and the double logarithmic axes in (c).

parameters (e.g., $\max (k)=133$ for $\gamma \sim 2.4$ as compared to $\max (k)=23$ for $\gamma \sim 4.0)$. While previous research on uncorrelated scale-free topologies has shown that genetic information disseminates rapidly when favorable alleles are strategically placed in such highly connected vertices [6], this high connectivity also makes these vertices more difficult to invade. Consider the case where the initial high fitness individual is placed in a vertex with low connectivity, which neighbors a high degree "hub" of degree $k$. Under the uniform selection mechanism previously described, it will take on average $k$ generations for the hub to adopt the high fitness value of its neighboring vertex. Therefore, increasing the degree of a vertex increases the average amount of time required for a neighboring vertex to invade it. In uncorrelated scale-free topologies under uniform selection, a single structural property thus simultaneously inhibits and facilitates the dissemination of information, depending on the degree of the vertex of initial placement.

The second factor influencing the decrease in $E[T]$ as a function of $\gamma$ is the distribution of path lengths $\left(L_{i}\right)$. As shown in Figure 2, uncorrelated scale-free topologies with larger scaling parameters were found to possess more homogeneous distributions of path lengths (e.g., $\gamma \sim 4.0$, Figure $2 \mathrm{a}$ ) than those with smaller scaling parameters (e.g., $\gamma \sim 3.4$ and $\gamma \sim 2.4$, in Figures $2 \mathrm{~b}$ and $2 \mathrm{c}$, respectively). As the scaling parameter is decreased, the distribution of path lengths becomes more heavily skewed, deviating from a normal distribution for all $\gamma<4.0\left(p<<0.01, \chi^{2}\right.$ test). Thus, the longer path lengths inherent in the topologies with smaller scaling parameters serve to slow the spread of information throughout the population.

The change in the variability of $T$ (Figure 1a), and consequently $E[T]$ (Figure 1b), as a function of $\gamma$ can be understood as follows. Scale-free topologies with lower scaling parameters possess larger variability in their degree distribution (e.g., compare the inset of Figure 2c with that of Figure 2a) and takeover time is (nonlinearly) inversely correlated with the degree of the vertex in which the initial high fitness individual is placed (Figure 1c). Since in our experiments the initial high fitness individuals are placed in each node of the population structure, one at a time, and
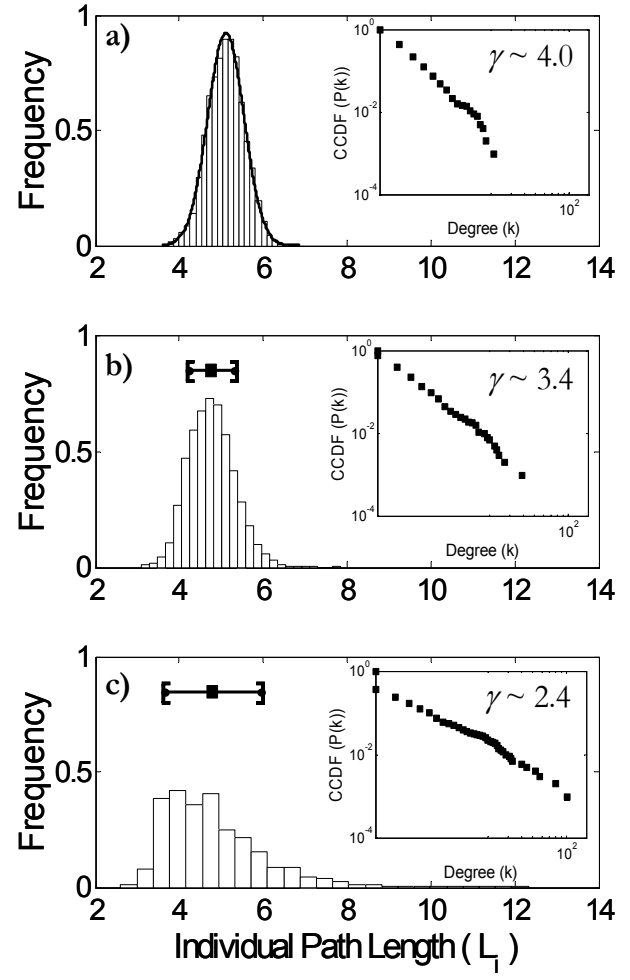

Figure 2. Frequency distributions of individual path lengths $\left(L_{i}\right)$ for (a) $\gamma \sim 4.0$, (b) $\gamma \sim 3.4$, and (c) $\gamma \sim 2.4$. Each distribution represents the frequency with which individual path lengths were observed across all ten graph instances. In (a), the distribution of path lengths is normally distributed $\left(p>0.01, \chi^{2}\right.$ test) and the solid line depicts a Gaussian PDF created using the sample mean and standard deviation of the individual path lengths. In (b) and (c), the solid squares denote the characteristic path length $(L)$, averaged across all ten graph instances, and the horizontal error bars denote one standard deviation across the graph instances. The insets depict the CCDF of the respective degree distributions $(P(k))$. 


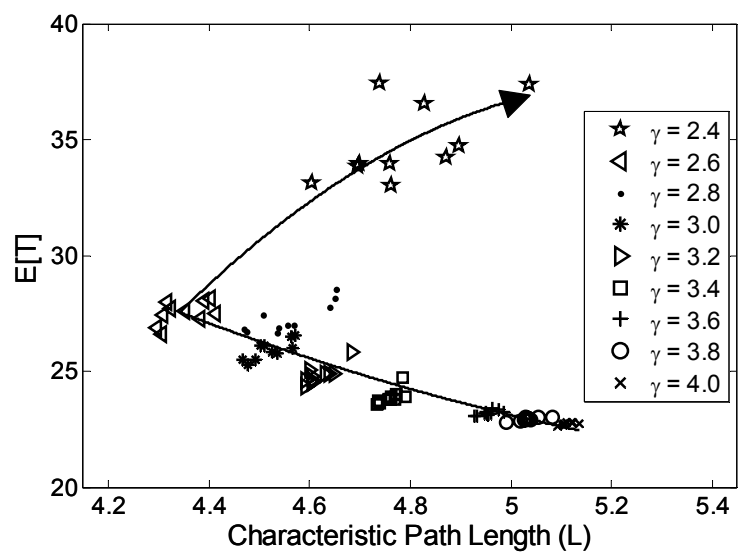

Figure 3. Average takeover time $(E[T]$, in generations) as a function of characteristic path length $(L)$ in uncorrelated $(r=$ 0) scale-free topologies. Each data point represents the average takeover time observed on a single graph instance. The crooked arrow is merely provided as a visual aid to indicate the surprising changes in $E[T]$ and $L$ as the scaling exponent decreases from $\gamma \sim 4.0$ to $\gamma \sim 2.4$.

topologies with smaller $\gamma$ have larger variation in the degree of the vertices in which the initial high fitness individuals are placed, the variability in $T$, and consequently in $E[T]$, increases.

In Figure 3, we show the relationship between characteristic path length $(L)$ and $E[T]$ over a range of scaling parameters $(\gamma)$. As this metric captures the average distance between all pairs of vertices in an interaction network, $L$ strongly influences the rate of information flow throughout many systems. For example, expected takeover times in regular population structures are known to increase linearly in $L$ [24]. Interestingly, as the scaling parameter decreases from $\gamma \sim 4.0$ ( $\times$ symbols $)$ to $\gamma \sim 2.6(\triangleleft$ symbols), $L$ decreases from an average of 5.1 to 4.3 , with a corresponding increase in $E[T]$ from 22.7 to 27.5 . In contrast, as $\gamma$ decreases from 2.6 to 2.4 (star symbols), the trend changes abruptly, with $L$ and $E[T]$ simultaneously increasing to 4.8 and 34.8 , respectively. This finding has two interesting implications. First, contrary to previous belief [21], the expected takeover time can actually decrease as the characteristic path length increases. Second, as witnessed by the topologies with $\gamma \sim 3.4$ (Figure 3, $\square$ symbols; Figure 2b, horizontal error bars) and $\gamma \sim 2.4$ (Figure 3, open stars; Figure 2c, horizontal error bars), interaction networks with statistically indistinguishable characteristic path lengths ( $p=$ 0.46 , unpaired t-test for distributions with unequal variance) can have significantly different expected takeover times $(p<<0.01$, unpaired t-test for distributions with unequal variance). As $L$ is an averaged metric, it belies the true nature of the underlying path length distribution (Figures 2b,c) and is therefore not wholly indicative of $E[T]$ in heterogeneous interaction topologies.

\subsection{Influence of Assortativity Alone}

We now consider the influence of assortativity on takeover times in scale-free topologies with $\gamma \sim 3$, as this scaling parameter is representative of graphs generated using the $\mathrm{AB}$ algorithm, which is commonly employed in studies of dynamical processes on scale-free networks (e.g., [20][25]). Figure 4a shows a general increase in the mean and variance of the expected takeover time $E[T]$ as assortativity $(r)$ increases from $r=-0.2$ to $r=0.2$. The heteroskedacticity of $E[T]$ across the values of assortativity considered herein results from an increase in the variability of the path length distribution as $r$ increases $\left(\sigma_{\mathrm{L}}{ }^{2}\right.$ increases linearly from 0.17 at $r=-0.2$ to 0.79 at $\left.r=0.2, \mathrm{R}^{2}=0.96\right)$, similar to the effect of decreasing $\gamma$ in uncorrelated scale-free topologies (Figure 2). The increase in $E[T]$ as a function of $r$ results from the nature of assortative networks, as explained below.

As assortativity increases, vertices of similar degree begin to connect to one another more frequently. This has two relevant implications. First, as the highly connected vertices attach to one another, the efficacy with which these vertices disseminate information is mitigated by their neighboring hubs, which are more difficult to invade than the low degree vertices that are typically adjacent to a hub in disassortative scale-free topologies. Second, as vertices of low connectivity begin to attach to one another, they form long linear chains across which information travels slowly. In contrast, more disassortative topologies promote the linking of dissimilar nodes (in terms of degree), facilitating the rapid promulgation of information by hubs and inhibiting the presence of long linear chains. Thus, the selective pressures
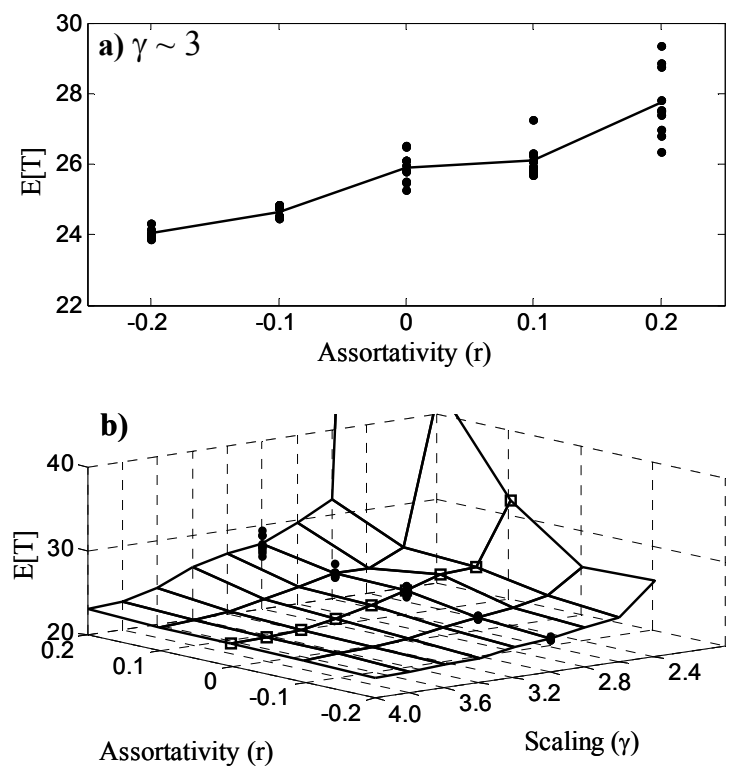

Figure 4. (a) Average takeover time ( $E[T]$, in generations) is shown as a function of assortativity $(r)$ in scale-free topologies with $\gamma \sim$ 3.0. Each data point represents the average takeover times on each graph instance; the solid line is drawn through the means of the data. (b) Average takeover time $(E[T]$, in generations) is presented as a surface function of assortativity $(r)$ and scaling $(\gamma)$. Each point in the surface is calculated as the average takeover time across all graph instances for a given combination of $r$ and $\gamma$. For reference, the data presented in Figure 4a (• symbols) and Figure 1b ( $\square$ symbols) are indicated in (b) to show how these figures relate to one another. Note that the vertical axis in (b) is truncated at 40 generations, down from the peak value of 132 generations, in order to scale the plot to better elucidate the shape of the nonlinear interaction in the response of $E[T]$ to $r$ and $\gamma$. 

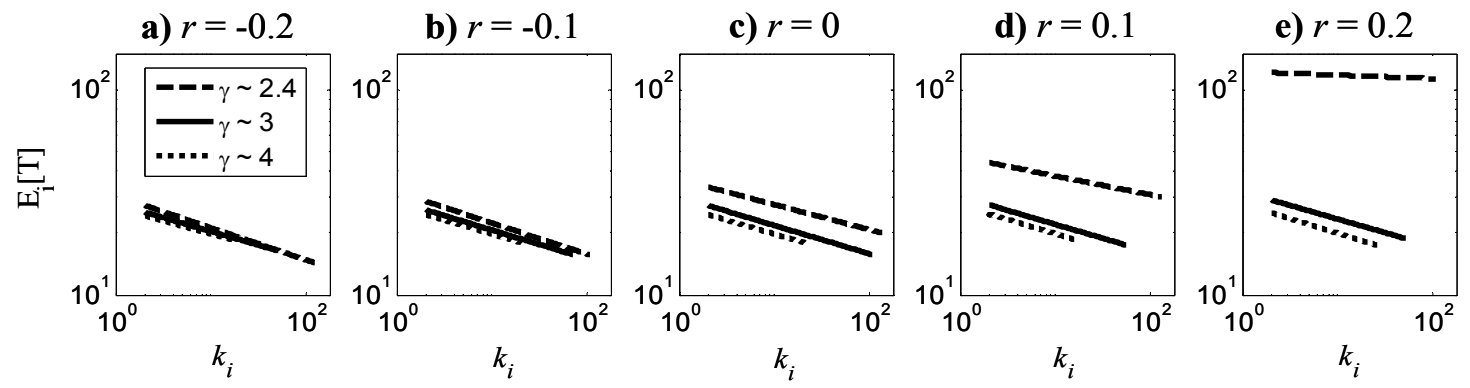

Figure 5. Each plot shows the average takeover time $E_{i}[T]$ as a function of the degree of the vertex in which the initial high fitness individual was placed $\left(k_{i}\right)$. Raw data has been substituted with best fit lines in order to indicate trends (see Figure 1c to get a sense of the variability around these lines). Assortativity increases from left to right from (a) $r=-0.2$ to (e) $r=0.2$. Note the log-log scale on all plots.

induced by scale-free population structures are easily tunable through the simple alteration of assortativity.

\subsection{Combined Scaling and Assortativity}

We now analyze the combined influence of scaling and assortativity on takeover times in scale-free topologies. In Figure $4 \mathrm{~b}$, we show the average takeover time $E[T]$ as a function of the scaling parameter $(\gamma)$ and assortativity $(r)$. The observed relationship is highly nonlinear. While $E[T]$ grows roughly linearly as $r$ increases for $3.8 \leq \gamma \leq 4.0\left(\mathrm{R}^{2}>0.88\right)$, the effect of assortativity becomes increasingly pronounced for $\gamma \leq 3.6$, with $E[T]$ rising sharply as the graph becomes more assortative. Similarly, for disassortative and uncorrelated graphs $(r \leq 0), E[T]$ grows approximately linearly as $\gamma$ decreases from $\gamma \sim 4.0$ to $\gamma \sim 2.6$ $\left(\mathrm{R}^{2}>0.91\right)$, after which an abrupt jump in $E[T]$ is observed for $\gamma \sim$ 2.4. For assortative graphs $(r>0), E[T]$ becomes more sensitive to lower values of $\gamma$. In combination, low values of the scaling parameter $(\gamma)$ and high values of assortativity $(r)$ give rise to exceptionally high takeover times; e.g., for $\gamma \sim 2.4$ and $r=0.2$ the maximum takeover time observed in a single simulation $(T)$ was 280 generations, with an average takeover time $E[T]$ of 132 generations. As a baseline for comparison, the average takeover times reported in [21] in same sized panmictic and 2D latticestructured populations were 14.83 and 44.15 generations, respectively.

In Figure 5, we show the average takeover time on graphs with $\gamma$ $\in\{2.4,3.0,4.0\}$, given that the single initial high fitness individual is placed in node $i\left(E_{i}[T]\right)$, as a function of the degree of this initial vertex $\left(k_{i}\right)$ and the assortativity $(r)$ of the graph. This relationship is also highly nonlinear; in disassortative graphs $(r<0$; Figures $5 \mathrm{a}, \mathrm{b})$, the scaling parameter has only a marginal effect on the rate of decay of $E_{i}[T]$ in $k_{i}$ and its overall magnitude. However, as assortativity increases ( $r \geq 0$; Figures $5 \mathrm{c}-\mathrm{e}), E_{i}[T]$ as a function of $k_{i}$ becomes increasingly sensitive to the scaling parameter (e.g., compare the dashed lines in Figures 5a-e), indicating that the influence of the degree of the vertex of initial placement on average takeover time is contingent upon the assortativity of the topology. Further, the rate of decay of $E_{i}[T]$ as a function of $k_{i}$ decreases dramatically at high assortativity and low scaling. For example, for graph instances with $\gamma \sim 2.4$ and $r=0.2$ (Figure 5e, dashed line), $E_{i}[T]$ is relatively invariant as a function of $k_{i}$. This finding has the interesting implication that in highly assortative scale-free graphs with low scaling parameters, the degree of the vertex in which the initial high fitness individual is placed has virtually no impact on the resulting takeover time, a result that rests in stark contrast to the relationship between $E_{i}[T]$ and $k_{i}$ observed in uncorrelated and disassortative scale-free topologies (Figures 5a-c).

We now turn our attention to the characteristic path length $(L)$ and the metric $\zeta$ presented in [21], and their relationships to average takeover times $(E[T])$, as the scaling parameter $(\gamma)$ and assortativity $(r)$ are varied. Note that the values of $\gamma$ are not explicitly shown in Figure 6 as they are in Figure 3; however, to better elucidate trends in the data, we provide lines as visual aids in each panel, and $\gamma$ decreases monotonically as these lines are read from bottom to top (e.g., compare the $r=0$ data in Figure 6c with the same data in Figure 3, where $\gamma$ is shown explicitly). In Figures 6a,c, we depict the relationship between $L$ and $E[T]$ as the graph instances are changed from disassortative $(r=-0.2$; Figure $6 \mathrm{a}, \mathrm{c}$, lines indicated by symbol) to uncorrelated ( $r=0$; Figure 6a,c, lines indicated by $\mathbf{0}$ symbol) to assortative ( $r=0.2$; Figure 6a,c, lines indicated by + symbol). In graph instances with $r=-0.2, E[T]$ decreases as a monotonic function of $L$. However, as the graph instances become more assortative, the relationship between $E[T]$ and $L$ exhibits an interesting shift. Consider the case of $\gamma \sim 2.6$ (Figure 6c, open circles). As these graph instances are tuned from disassortative ( $r=$ $0.2)$ to uncorrelated $(r=0)$, the characteristic path length remains unchanged $(p=0.34$, unpaired t-test for distributions with unequal variance), while the average takeover time increases significantly ( $p$ $<<0.01$, unpaired t-test for distributions with unequal variance). As these graph instances are further tuned from uncorrelated to assortative $(r=0.2)$, both $L$ and $E[T]$ increase significantly ( $p<<$ 0.01 , unpaired t-test for distributions with unequal variance). This has the interesting implication that, while for highly disassortative graphs average takeover time $(E[T])$ is negatively correlated with characteristic path length $(L)$, the relationship between $L$ and $E[T]$ becomes an ambiguous multifunction as assortativity increases, with significantly different average takeover times observed on graph instances possessing indistinguishable characteristic path lengths.

In Figures $6 \mathrm{~b}, \mathrm{~d}$ (note the log-log scaling), we depict the relationship between $\zeta$ and $E[T]$. For uncorrelated and assortative graphs (lines indicated by $\mathbf{0}$ and + symbols, respectively), $E[T]$ and $\zeta$ were found to be strongly correlated $\left(\mathrm{R}^{2}=0.94\right.$ for $r=0, \mathrm{R}^{2}=0.88$ for $r=0.2$, between $\log (E[T])$ and $\log (\zeta))$, with $E[T]$ growing sublinearly in $\zeta$ 
a)

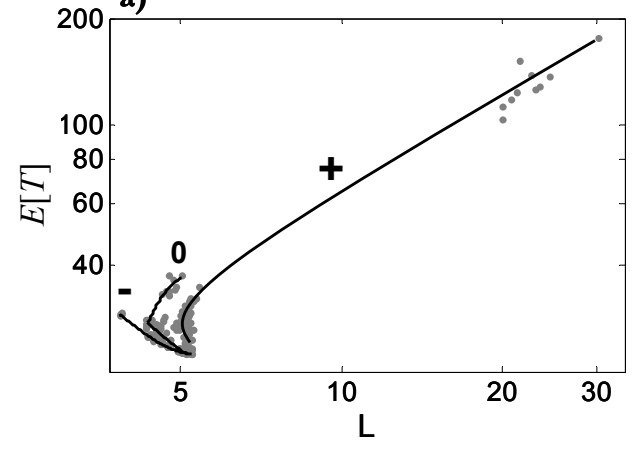

c)

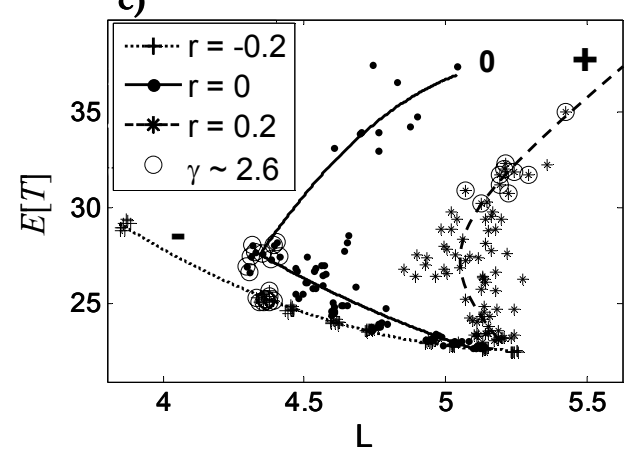

b)

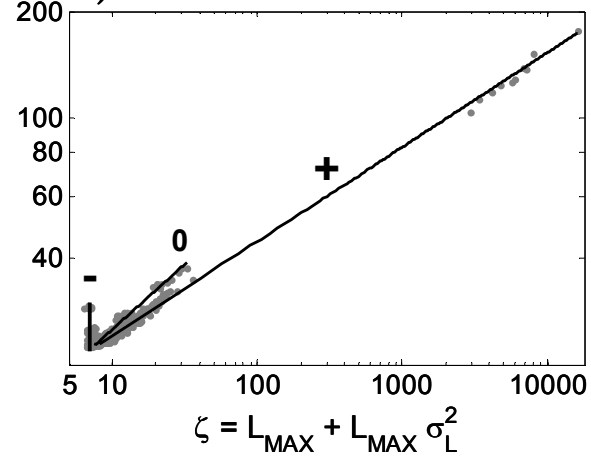

$\left.10^{1.6} \mathbf{d}\right)$

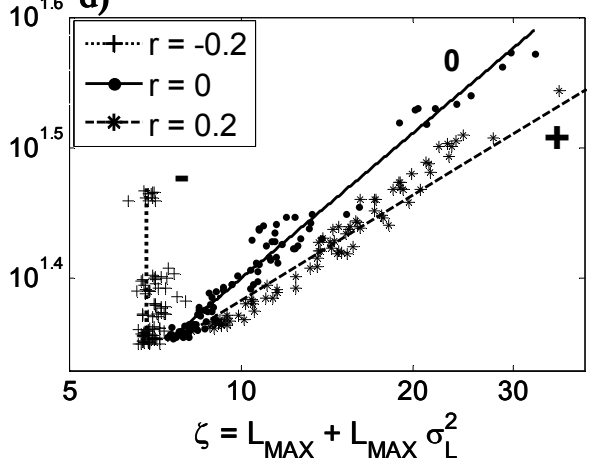

Figure 6. (a) Average takeover time $(E[T])$ as a function of characteristic path length $(L)$ and (b) $E[T]$ as a function of the empirical metric provided in [21] $\left(\zeta=L_{M A X}+L_{M A X} \sigma_{L}^{2}\right)$. The data shown in (c) and (d) are magnifications of the lower regions of the data presented in (a) and (b), respectively. Each data point represents the average takeover time observed on a single graph instance. In (c), the circle symbol (indicating the data corresponding to $\gamma \sim 2.6$ ) is used to show how data for a single scaling exponent "turns the corner" in this unusual relationship between $E[T]$ and $L$ as assortativity increases. The lines are added as a visual aid to indicate trends in the data. The negative (-), zero (0), and positive $(+)$ symbols are also included as a visual aid to indicate the sign of the assortativity. Note that the data for $r=0$ in (c) is the same as that presented in Figure 3. Axes are scaled logarithmically in $(a, b, d)$ and linearly in (c).

over many orders of magnitude. However, the slope of the relationship between $\log (E[T])$ and $\log (\zeta)$ increases as assortativity decreases, to the point where the line is essentially vertical at $r=$ 0.2 and thus there is no predictive power in $\zeta$ for disassortative graphs $\left(\mathrm{R}^{2} \sim 3 \mathrm{e}-4\right)$. These observations have several interesting implications. First, $\zeta$ is not a useful predictor of average takeover times $(E[T])$ in disassortative graphs $(r<0)$. Second, while $\zeta$ is highly predictive of $E[T]$ in uncorrelated and assortative graphs ( $r \geq$ $0)$, this relationship is sublinear, in contrast to the observations made in [21]. Third, even in uncorrelated and assortative graphs, the relationship between $E[T]$ and $\zeta$ is contingent upon the degree of assortativity, a factor that was not considered in [21].

\section{SUMMARY AND CONCLUSIONS}

The results of this study demonstrate that the scaling parameter $(\gamma)$ and the degree of assortativity $(r)$ strongly affect the flow of advantageous alleles throughout scale-free population structures, and thus impact the selective pressures induced by these topologies. Specifically, our results demonstrate that decreasing $\gamma$ increases the average takeover time because (a) as $\gamma$ decreases, the distribution of vertex connectivity spans a greater domain, possessing highly connected vertices that are difficult to invade, and (b) the distribution of path lengths becomes more heavily skewed, such that information must travel across greater distances to fully saturate the population. Increasing assortativity $(r)$ increases the average takeover time because (a) highly connected vertices are likely to attach to one another, therefore limiting one another's influence and (b) as vertices of low connectivity link together, they form long linear chains of nodes over which information travels slowly. In disassortative and uncorrelated topologies, the average takeover time was found to decay faster than linearly as a function of the degree of the vertex in which the initial high fitness individual was placed, and the magnitude of this relationship was found to be a function of $\gamma$. In contrast, in scale-free population structures with low $\gamma$ and high $r$, the average takeover time was virtually independent of the degree of the vertex in which the initial high fitness individual is placed. Thus, the influence of the degree of the vertex of initial placement is contingent upon both assortativity and the scaling parameter of the degree distribution. In combination, the scaling parameter and degree of assortativity were found to exhibit a nonlinear influence on the average takeover time, with the highest takeover times observed on highly assortative graphs possessing low scaling parameters. 
In disassortative scale-free graphs, the average takeover time was a nonlinear inverse function of the characteristic path length $(L)$, but was virtually independent of the metric $\zeta$ suggested in [21] to be predictive of average takeover times. Conversely, in uncorrelated and assortative scale-free topologies, average takeover time was a sublinear function of $\zeta$, but was an ambiguous multifunction of $L$. This result suggests that any general topological metric designed to predict average takeover times will have to incorporate assortativity. Future work will investigate how these relationships are affected by population size.

The uniform selection method considered in this study is clearly a simplification of the selection operators commonly used in evolutionary algorithms. However, even in this simplified model of information flow, non-trivial relationships were observed between the topological properties of population structure and average saturation time. Our results may serve as a useful frame of reference in a variety of models of dynamical processes on networks, such as graph-based models of disease transmission or fad proliferation. For example, it may be interesting to investigate the impact of scaling and assortativity in binary decision models that utilize heterogeneous threshold-based update policies (e.g., [29]), in which the decision of any single individual is contingent upon both the individual's particular response function and the decisions of neighboring individuals. Future work will seek to address the inclusion of this update policy as well as others, such as the frequency-dependent selection mechanisms commonly employed in studies of evolutionary games (e.g., [25][28]).

While lattice-based interaction networks have been explored for evolutionary optimization algorithms (e.g., see [2] and references therein), the potential utility of scale-free population structures is still unclear. Future work will seek to elucidate how, if at all, one can exploit scale-free population structures to improve the search performance of population-based optimization algorithms. For example, we hypothesize that it may be possible to adaptively control selective pressure in scale-free population structures by dynamically changing assortativity through simple swaps.

\section{References}

[1] Albert, R., Jeong, H., \& Barabàsi, A.L. Diameter of the WorldWide Web. Nature, 401 (1999), 130-131.

[2] Alba, E., \& Dorronsoro, B. The exploration/exploitation tradeoff in dynamic cellular genetic algorithms. IEEE Transactions on Evolutionary Computation, 9, 2 (2005), 126-142.

[3] Barabàsi, A.L. \& Albert, R. Emergence of scaling in random networks. Science, 286 (1999), 509-512.

[4] Clauset, A., Shalizi, C.S., \& Newman, M.E.J. Power-law distributions in empirical data. arXiv:0706.1062v1, (2007).

[5] Ebel, H., Mielsch, L., \& Bornholdt, S. Scale-free topology of email networks. Physical Review E, 66 (2002), 035103(R).

[6] Giacobini, M., Tomassini, M., \& Tettamanzi, A. Takeover time curves in random and small-world structured populations. In Proc. Genetic and Evolutionary Computation Conference. ACM Press, New York, NY, 2005, 1333-1340.

[7] Giacobini, M., Tomassini, M., Tettamanzi, A., \& Alba, E. Selection intensity in cellular evolutionary algorithms for regular lattices. IEEE Transactions on Evolutionary Computation, 9, 5 (2005), 489-505.

[8] Goldberg, D.E., \& Deb, K. A comparative analysis of selection schemes used in genetic algorithms. In Proc. Foundations of Genetic Algorithms. Morgan-Kauffman, San Mateo, CA, 1991, 6993.
[9] Gómez-Gardeñes, J., \& Moreno, Y. From scale-free to ErdosRényi networks. Physical Review E, 73 (2006), 056124.

[10] Gorges-Schleuter, M. An analysis of local selection in evolution strategies. In Proc. Genetic and Evolutionary Computation Conference. Morgan Kaufmann, San Francisco, CA, 1999, 847854.

[11] Hauert, C., \& Doebeli, M. Spatial structure often inhibits the evolution of cooperation in the snowdrift game. Nature, 428 (2004), 643-646.

[12] Jeong, H., Mason, S.P., Barabàsi, A.L., \& Oltvai, Z.N. Lethality and centrality in protein networks. Nature, 411 (2001), 41-42.

[13] Keeling, M. J. The effects of local spatial structure on epidemiological invasions. Proceedings of the Royal Society of London B, 266, (1999), 859-867.

[14] Krapivsky, P.L., Redner, S., \& Leyvraz, F. Connectivity in growing random networks. Physical Review Letters, 85, 21 (2000), 4629.

[15] Liljeros, F., Edling, C.R., Amaral, L.A.N., Stanely, H.E., \& Åberg, Y. The web of human sexual contacts. Nature, 411 (2001), 907908.

[16] Maslov, S., \& Sneppen, K. Specificity and stability in topology of protein networks. Science, 296 (2002), 910-913.

[17] Motter, A.E., de Moura, A.P.S., Lai, Y.C., \& Dasgupta, P. Topology of the conceptual network of language. Physical Review E, 65 (2002), 065102(R).

[18] Newman, M.E.J. Assortative mixing in networks. Physical Review Letters, 89, 20 (2002), 208701.

[19] Nowak, M.A., \& May, R.M. Evolutionary games and spatial chaos. Nature, 359 (1992), 826-829.

[20] Pastor-Satorras, R. \& Vespignani, A. Epidemic spreading in scalefree networks. Physical Review Letters, 86, 14 (2001), 3200.

[21] Payne, J.L., \& Eppstein, M.J. Takeover times on scale-free topologies. In Proc. Genetic and Evolutionary Computation Conference. ACM Press, New York, NY, 2007, 308-315.

[22] Poncela, J., Gómez-Gardeñes, J., Floría, L.M., \& Moreno, Y. Robustness of cooperation in the evolutionary prisoner's dilemma on complex networks. New Journal of Physics, 9 (2007), 184.

[23] Rong, Z., Li, X., \& Wang, X. Roles of mixing patterns in cooperation on a scale-free networked game. Physical Review E, 76 (2007), 027101.

[24] Rudolph, G. On takeover times in spatially structured populations: array and ring. In Proc. 2nd Asia-Pacific Conference on Genetic Algorithms and Applications. Global-Link Publishing Company, Hong Kong, 2000, 144-151.

[25] Santos, F.C., \& Pacheco, J.M. Scale-free networks provide a unifying framework for the emergence of cooperation. Physical Review Letters, 95, 9 (2005), 098104.

[26] Sarma, J., \& De Jong, K. An analysis of the effect of the neighborhood size and shape on local selection algorithms. In Proc. Parallel Problem Solving from Nature Conference. SpringerVerlag, Heidelberg, 1996, 236-244.

[27] Sayama, H., Kaufman, L., \& Bar-Yam, Y. Spontaneous pattern formation and genetic diversity in habitats with irregular geographical features. Conservation Biology, 17 (2003), 893-900.

[28] Smith, J.M. Evolution and the Theory of Games. Cambridge University Press, Cambridge, UK, 1982.

[29] Watts, D.J. A simple model of global cascades on random networks. PNAS, 99, 9 (2002), 5766-5771.

[30] Xulvi-Brunet, R., \& Sokolov, I.M. Reshuffling scale-free networks: from random to assortative. Physical Review E, 70 (2004), 066102. 